\title{
Quantum-to-classical transition in the work distribution for chaotic systems
}

\author{
Ignacio García-Mata, ${ }^{1,2}$ Augusto J. Roncaglia, ${ }^{3}$ and Diego A. Wisniacki ${ }^{3}$ \\ ${ }^{1}$ Instituto de Investigaciones Físicas de Mar del Plata (IFIMAR), CONICET-UNMdP, Funes 3350, B7602AYL Mar del Plata, Argentina \\ ${ }^{2}$ Consejo Nacional de Investigaciones Científicas y Tecnológicas (CONICET), Godoy Cruz 2290 (C1425FQB) CABA, Argentina \\ ${ }^{3}$ Departamento de Física "J. J. Giambiagi” and IFIBA, FCEyN, Universidad de Buenos Aires, 1428 Buenos Aires, Argentina
}

(Received 3 November 2016; published 12 May 2017)

\begin{abstract}
The work distribution is a fundamental quantity in nonequilibrium thermodynamics mainly due to its connection with fluctuation theorems. Here, we develop a semiclassical approximation to the work distribution for a quench process in chaotic systems that provides a link between the quantum and classical work distributions. The approach is based on the dephasing representation of the quantum Loschmidt echo and on the quantum ergodic conjecture, which states that the Wigner function of a typical eigenstate of a classically chaotic Hamiltonian is equidistributed on the energy shell. Using numerical simulations, we show that our semiclassical approximation accurately describes the quantum distribution as the temperature is increased.
\end{abstract}

DOI: 10.1103/PhysRevE.95.050102

During the last years there was an increasing interest in the field of thermodynamics and statistical physics [1,2]. This rebirth of the area has been fueled by the technological advances that lead to control with extreme precision the dynamics of small quantum systems. In the context of nonequilibrium statistical physics, standard concepts such as work and heat are random variables characterized by a distribution, and have been redefined so as to include quantum effects [3-5]. Notably, fluctuation theorems, such as Jarzynski [6] and Crooks [7] equalities, have been extended to the quantum regime. Despite the similarities between standard and quantum fluctuation theorems, the transition between quantum and classical descriptions seems to be elusive, and this difference resides in the quantum definition of work.

The accepted definition of quantum work, performed or extracted after a single realization of a process in an isolated system, can be formulated in terms of two projective measurements of the system's energy or two-point measurement [3-5]. Thus defined, the fluctuations in the value of work have thermal and quantum origins. However, it could also be argued that this definition is arbitrarily proposed so that the fluctuation relations are fulfilled. It has been thus suggested [8,9] that a justification of this definition would be to test the correspondence principle in the classical limit. Several studies have considered the quantum and classical distributions of work in harmonic systems [10-15], where analytical solutions are available. Interestingly, in Ref. [8], by employing a semiclassical method, it has been shown that in one-dimensional integrable systems (quartic oscillator) there is a correspondence between classical and quantum transition probabilities. For chaotic systems, there is some numerical evidence showing that the correspondence principle also applies [9], supporting the quantum definition of work.

Here, we go a step further: We present a semiclassical expression for the distribution of work done on a system after a quench for fully chaotic system. We show analytically that the correspondence between the classical and quantum distributions is recovered from the semiclassical expression in the limit of vanishing Planck constant. Furthermore, we verify the good agreement between the semiclassical and quantum work distributions, and the quantum-classical correspondence, using numerical simulations of a quantum particle inside a stadium billiard that suddenly changes its inner potential. The main ingredients we used to derive the semiclassical expression are the connection of the characteristic function with the fidelity amplitude or Loschmidt echo [16], the semiclassical dephasing representation [17-19] of the fidelity amplitude, and the conjecture by Berry and Voros that the Wigner functions for eigenstates of chaotic systems are peaked on the corresponding energy shell [20,21]. Both approximations have proven to be very useful separately to understand the key features of generic quantum chaotic systems.

We consider the following process applied to a quantum system described by a Hamiltonian $H_{\xi}$ that depends on a control parameter $\xi$. We assume that the system is in thermal equilibrium with a bath at inverse temperature proportional to $\beta^{-1}$ (in the following, we will consider $k_{B}=1$ ). The initial state of the system is then $\rho_{\beta}=\exp \left(-\beta H_{\xi_{0}}\right) / Z_{\xi_{0}}^{Q}$ and $Z_{\xi_{0}}^{Q}=\operatorname{Tr}\left[\exp \left(-\beta H_{\xi_{0}}\right)\right]$. Next, the system is decoupled from the bath, and the control parameter is suddenly changed from $\xi_{0}$ to $\xi_{f}$, taking the system away from equilibrium. As a result, the work performed on the system after the quench is a random quantity given by the difference of the outcomes of two energy measurements $W=E_{\xi_{f}}^{n}-E_{\xi_{0}}^{m}$, one at the beginning and the other at the end of the process. In this way, work is characterized by the following distribution [3-5],

$$
P^{Q}(W)=\sum_{n, m} P^{Q}(m) P^{Q}(n \mid m) \delta\left[W-\left(E_{\xi_{f}}^{n}-E_{\xi_{0}}^{m}\right)\right],
$$

where $P^{Q}(m)=e^{-\beta E_{\xi_{0}}^{m}} / Z_{\xi_{0}}^{Q}, P^{Q}(n \mid m)$ is the quantum conditional probability to obtain the eigenstate of $E_{\xi_{f}}^{n}$ at the final measurement, given that the initial result was $E_{\xi_{0}}^{m}$ (for a quench this is equal to the squared overlap between the corresponding eigenstates), and $\delta$ is the Dirac $\delta$ function. Different strategies that enable the reconstruction of the quantum work distribution have been put forward [22-25] and also recently measured, verifying fluctuation theorems in the quantum regime [26,27].

In our approach we consider the characteristic function $[5,10]$ that is defined as the Fourier transform of the work distribution

$$
G(u)=\int d W e^{i u W} P(W)
$$


Notably, the characteristic function can be directly measured using interferometric techniques, as proposed in Refs. [23,24] and implemented experimentally in Ref. [26]. As it was noticed in Ref. [16], the characteristic function for a quantum system that is subjected to a quench can be expressed as

$$
G^{Q}(u)=\left\langle e^{i u H_{\xi_{f}}} e^{-i u H_{\xi_{0}}}\right\rangle=\operatorname{Tr}\left[e^{i u H_{\xi_{f}}} e^{-i u H_{\xi_{0}}} \rho_{\beta}\right],
$$

where $\rho_{\beta}$ is a thermal state of $H_{\xi_{0}}$ at temperature $\beta^{-1}$. In this expression, $u$ is a timelike variable, so, if it is replaced by $t / \hbar$, Eq. (3) can be regarded as the average amplitude probability over two different time evolutions, or simply an averaged Loschmidt echo (LE) amplitude [28-30]. Remarkably, in Refs. [17-19] a very efficient semiclassical method to compute the LE amplitude — called dephasing representation (DR) was proposed. It is based on the initial value representation [31] and its success is partly due to the validity of the shadowing theorem [18]. Moreover, it circumvents some of the problems of the previous semiclassical techniques, such as the need for using two pairs of classical trajectories, the search for periodic orbits, or other special classical trajectories. The DR has been successfully used in various areas such as the study of irreversibility and the Loschmidt echo [17-19,32,33], the local density of states in chaotic systems [34], and molecular dynamics [35]. Below, we derive a semiclassical expression for the characteristic function that is based on the use of the DR.

Let us start the derivation by considering a quench, where a parameter of the Hamiltonian $H_{\xi}=H_{0}+\xi V$ is suddenly changed from $\xi_{0} \rightarrow \xi_{f}$. Then, the characteristic function for this process can be expressed in the DR as [19]

$$
G^{\mathrm{DR}}(u)=\int d^{2 D} x_{0} \mathcal{W}_{\beta}\left(x_{0}\right) \exp \left[i \Delta S\left(x_{0}, u \hbar\right) / \hbar\right],
$$

where $D$ is the number of degrees of freedom, $x \equiv(q, p) \in$ $\mathbb{R}^{2 D}$ is a collective notation, $\Delta S\left(x_{0}, t\right) \equiv \int_{0}^{t} d \tau \Delta H[x(\tau)]$ is an action difference, $\Delta H \equiv H_{\xi_{f}}-H_{\xi_{0}}, x(\tau)$ denotes the phase space coordinates at time $\tau$ of a trajectory generated by the Hamiltonian $H_{\xi_{0}}$ with the initial condition $x_{0}$, and $\mathcal{W}_{\beta}\left(x_{0}\right)$ is the Wigner function of the thermal state $\rho_{\beta}$.

Since the thermal state of the Hamiltonian $H_{\xi_{0}}$ is $\rho_{\beta}=$ $\sum_{m} e^{-\beta E_{\xi_{0}}^{m}}\left|E_{\xi_{0}}^{m}\right\rangle\left\langle E_{\xi_{0}}^{m}\right| / Z_{\xi_{0}}^{Q}$, where $\left|E_{\xi_{0}}^{m}\right\rangle$ are the eigenstates of $H_{\xi_{0}}$, its Wigner function can be written as a linear combination of the Wigner functions $\mathcal{W}_{m}$ of each eigenstate, $\mathcal{W}_{\beta}(x)=$ $\sum_{m} e^{-\beta E_{\xi_{0}}^{m}} / Z_{\xi_{0}}^{Q} \mathcal{W}_{m}(x)$. Now comes the key approximation that we use. We consider a quantum system with a fully chaotic classical counterpart, a system where the quantum ergodic conjecture (QEC) applies [20,21]. The QEC states that the Wigner function that represents a typical $E$-energy eigenstate of a classically chaotic Hamiltonian can be approximated by the classical Liouville probability density for the $E$-energy shell of the Hamiltonian,

$$
\mathcal{W}_{m}(x)=\frac{\delta\left[E_{\xi_{0}}^{m}-H_{\xi_{0}}(x)\right]}{\int d^{2 D} x \delta\left[E_{\xi_{0}}^{m}-H_{\xi_{0}}(x)\right]} .
$$

In addition, in order to simplify even more the final expression, we will consider that $[8,9] e^{-\beta E_{\xi_{0}}^{m}} / Z_{\xi_{0}}^{Q} \approx$ $\int_{E_{\xi_{0}}^{m}}^{E_{\xi_{0}}^{m+1}} e^{-\beta E} / Z_{\xi_{0}}^{C} g(E) d E$, where $Z_{\xi_{0}}^{C}=\int d x \exp \left[-\beta H_{\xi_{0}}(x)\right]$ is the classical partition function. Applying these
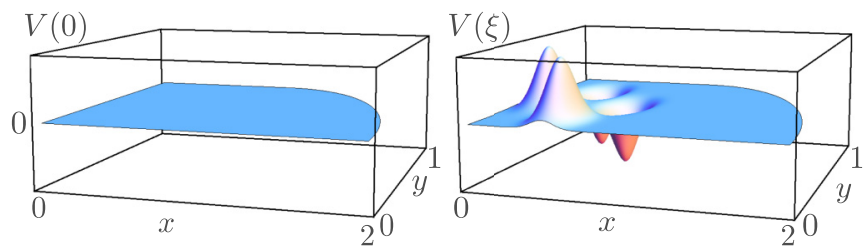

FIG. 1. Schematic depiction of the quench scheme: $H_{0} \rightarrow H_{0}+$ $V(\xi)$, where $H_{0}=\left(p_{x}^{2}+p_{y}^{2}\right)$. The Gaussians in the quench potential are centered at $\left(x_{1}, y_{1}\right)=(0.2,0.4),\left(x_{2}, y_{2}\right)=(0.67,0.5),\left(x_{3}, y_{3}\right)=$ $(0.5,0.15)$, and $\left(x_{4}, y_{4}\right)=(0.3,0.75)$.

approximations to Eq. (4), we arrive at the main result of this Rapid Communication, a semiclassical expression of the characteristic function (see Supplemental Material [36]),

$$
G^{S C}(u)=\int d^{2 D} x_{0} \frac{e^{-\beta H_{\xi_{0}}\left(x_{0}\right)}}{Z_{\xi_{0}}^{C}} \exp \left[i \Delta S\left(x_{0}, u \hbar\right) / \hbar\right] .
$$

It is worth pointing out that the classical work distribution $P^{C}(W)[8,37]$ can be recovered from $G^{S C}(u)$. This can be done by taking the limit $\hbar \rightarrow 0$ and applying the inverse Fourier transform to $G^{S C}(u)$ (see Supplemental Material [36]),

$$
P_{\hbar \rightarrow 0}^{S C}(W)=\frac{1}{2 \pi} \int d u e^{-i u W} G_{\hbar \rightarrow 0}^{S C}(u)=P^{C}(W) .
$$

So far, we have derived a semiclassical expression for the characteristic function using several approximations. In principle, this approach could be applied to fully chaotic systems; below, we test Eqs. (6) and (7) numerically for a specific model.

The system that we consider is a paradigmatic model of quantum chaos: a quantum particle inside a desymmetrized stadium billiard (with mass $m=\frac{1}{2}$, radius $r=1$, and straight line of length $l=1)$. Notice that in this case the hard walls of the billiard do not move in the process. In Refs. [9,38] it was numerically observed that for systems with moving hard walls, the quantum-classical correspondence depends strongly on the adiabaticity of the process. Furthermore, when the boundary is quenched infinitely fast, it is shown that there is no connection between quantum and classical distributions. Here, we consider a quench that consists of a sudden change of the Hamiltonian parameter, from $\xi_{0}=0$ to $\xi_{f}=85$, with a smooth potential given by four Gaussians: $V(\xi)=\xi \sum_{i=1}^{4}(-1)^{i-1} \exp \left(-\left[\left(x-x_{i}\right)^{2}-\right.\right.$ $\left.\left.\left(y-y_{i}\right)^{2}\right] /\left(2 \sigma^{2}\right)\right], \sigma=0.1$ their widths (see Fig. 1). The Gaussians are centered at $\left(x_{1}, y_{1}\right)=(0.2,0.4),\left(x_{2}, y_{2}\right)=(0.67,0.5)$, $\left(x_{3}, y_{3}\right)=(0.5,0.15)$, and $\left(x_{4}, y_{4}\right)=(0.3,0.75)$.

In order to obtain the quantum characteristic function $G^{Q}(u)$ and $P^{Q}(W)$, we first compute the eigenstates of the unperturbed stadium using the scaling method [39], and then we perform a diagonalization using the eigenbasis of the initial Hamiltonian $H_{\xi_{0}}$ to obtain the quenched system eigenstates. We have considered the first 2500 states, but the basis is truncated at 5600 (to ensure the accuracy of the first 2500). The semiclassical approximations of $G^{S C}(u)$ and $P^{S C}(W)$ are computed using Eq. (6) (and its Fourier transform) by sampling over a set of classical trajectories.

In Fig. 2, we show $P^{Q}(W)$ and $P^{S C}(W)$, where different values of temperature $\beta^{-1}$ are considered, and $\hbar=1$. In the 

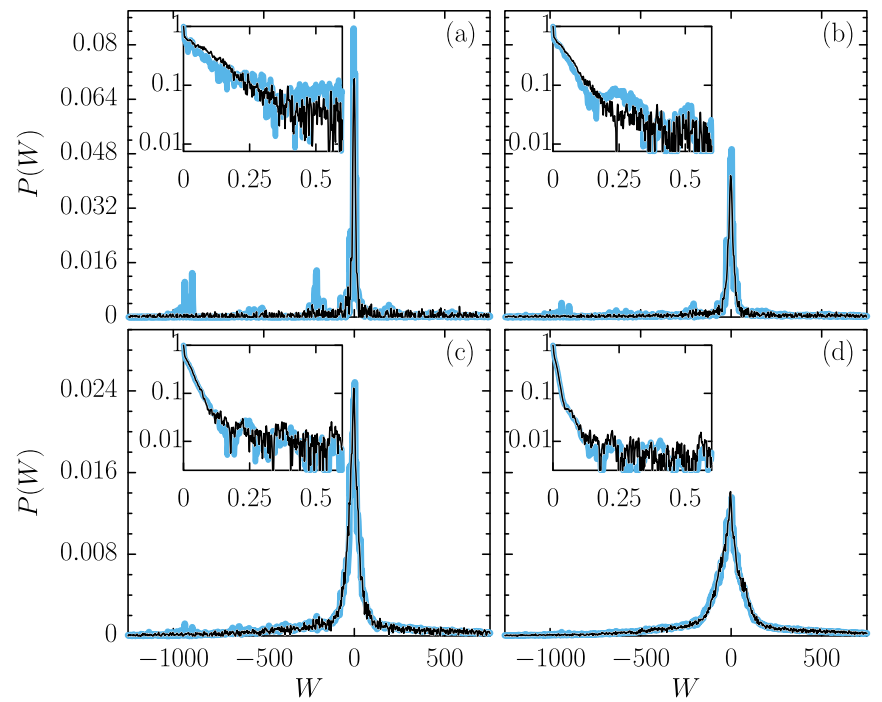

FIG. 2. Quantum (light blue/gray) and semiclassical (black) work distribution for different temperatures, from (a)-(d): $\beta=2^{-6}, 2^{-8}$, $2^{-10}, 2^{-12}$. In the inset we show the characteristic function for each temperature (the $y$ axis is in $\log _{10}$ scale).

inset we show the corresponding characteristic functions. The solid light-blue (gray) lines correspond to the quantum result and the black curves to the semiclassical one. We remark that the accuracy of the semiclassical calculations does not depend very much on the number of trajectories that we use [40]. In particular, the results shown in Fig. 2 were obtained using only $\approx 9 \times 10^{4}$ randomly chosen initial conditions. We can see that the main features of the work distribution are well reproduced for all these temperatures. In the case of $\beta=2^{-6}$ we can see that the semiclassical distribution deviates from the quantum one. This is due to the fact that the proportion of low-lying energy eigenstates contributing to the distribution is significant, making our approximation less accurate. If $\Delta E=$ $\left(4 \pi / \hbar^{2}\right) /(2 m A)$ is the mean level spacing of a quantum billiard of area $A$ and mass $m$, then for $\beta=2^{-6}$ the relevant number of states is $\sim 10$, so only a few, low-lying energies contribute. On the other hand, for smaller values of $\beta$, the agreement very good.

Some of the importance of $P(W)$ comes from the quantum fluctuation relations and the possibility, for example, to extract thermodynamical information such as the change in free energy, from the quantum Jarzynsky relation

$$
\Delta F \equiv-\frac{1}{\beta} \ln \frac{Z_{\xi_{f}}^{Q}}{Z_{\xi_{0}}^{Q}}=-\frac{1}{\beta} \ln \left\langle e^{-\beta W}\right\rangle,
$$

in systems out of equilibrium. Here, we use the Jarzynksy equality to test the accuracy of the semiclassical approximation. In Fig. 3, we show the evaluation of Jarzynski equaltity for a larger set of temperatures in the range $\beta^{-1} \in\left[2^{7}, 2^{13}\right]$ for both the quantum and semiclassical calculations. As expected, Fig. 2 shows that the agreement achieved by the semiclassical approximation is better as temperature increases.

In Figs. 2 and 3, we have demonstrated that our semiclassical method provides a good approximation of $P^{Q}(W)$ for a chaotic system as the temperature increases. Now we proceed

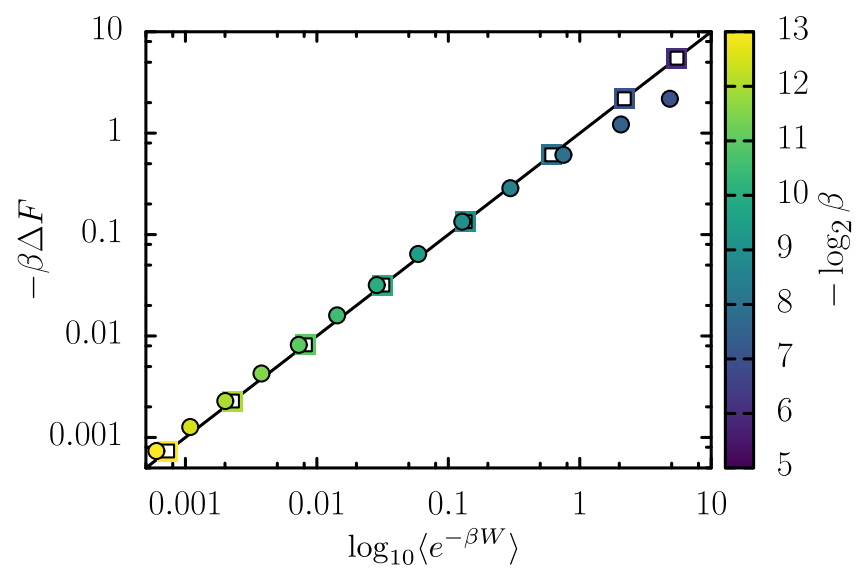

FIG. 3. Jarzinsky relation for the quenched described in the text. The squares correspond to the results obtained using the quantum work distribution, and the circles have been computed using the semiclassical approximation of the characteristic function for different temperatures. The scale of the $x y$ axis is $\log _{10}-\log _{10}$.

to show that the quantum-classical correspondence is achieved by taking the usual semiclassical limit $\hbar \rightarrow 0$ and comparing the resulting work distribution with its classical counterpart (see Supplemental Material [36]). For numerics, the classical work distribution is obtained by randomly sampling initial conditions in phase space, with the corresponding energies $E_{\xi_{0}}=p_{x}^{2}+p_{y}^{2}$. Then, from $H_{\xi_{f}}$ we evaluate the final energies $E_{\xi_{f}}$, and obtain the classical transition probabilities [8]. Finally, $P^{C}(W)$ is evaluated by considering a Boltzmann distribution of initial conditions. In the main panel of Fig. 4, we show $P^{S C}(W)$ for $\hbar=0.01,0.1,0.5,1$ and $P^{C}(W)$ (obtained using $4 \times 10^{6}$ initial conditions). In this case it is easy to see that the classical distribution of work is independent of the temperature, and as we increase the temperature and reduce the value of $\hbar$, the semiclassical distribution approaches the classical one. In the inset of Fig. 4, the corresponding characteristic function is shown for different $\hbar$ and, as expected, it can also be seen that $G^{S C} \stackrel{\hbar \rightarrow 0}{\longrightarrow} G^{C}$.

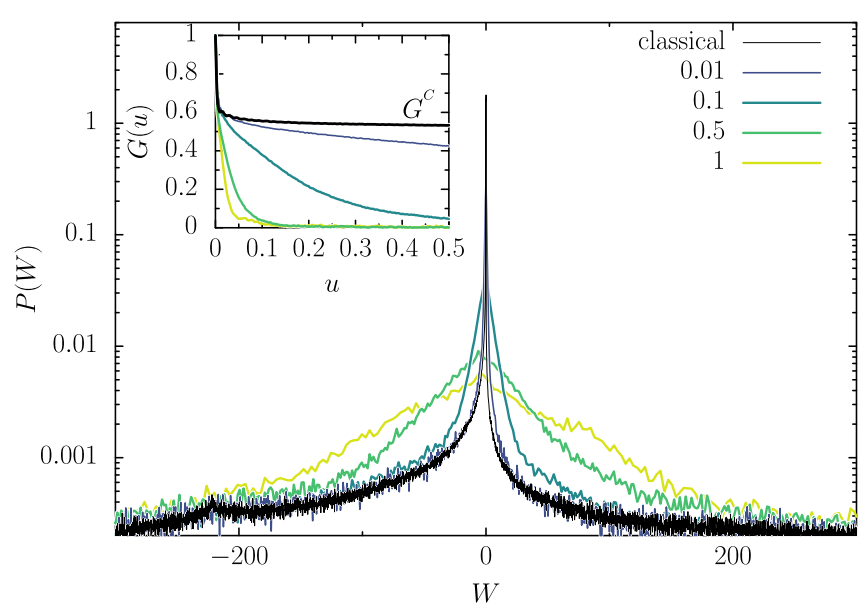

FIG. 4. Classical and semiclassical work distributions for $\beta=$ $2^{-12}$, and $\hbar=0.01,0.1,0.5,1$. Semiclassical results were obtained by the numerical evaluation of Eq. (6). The scale of the $y$ axis is $\log _{10}$. 
In summary, we have shown the quantum-to-classical correspondence of the work distribution for a sudden quench applied on a chaotic system. This was done using a semiclassical approach that provides a bridge between the quantum and classical domains. This approach is based on the study of the characteristic function that, in the quantum case, can be expressed as a quantum evolution. In this way, we developed a semiclassical expression for the characteristic function that relies on the dephasing representation and on the quantum ergodic conjecture, which states that the Wigner function of chaotic eigenfunctions are equidistributed over the corresponding energy shell. We have shown that the work distribution obtained from the semiclassical characteristic function is in good agreement with the quantum one over a wide range of temperatures, and its accuracy increases with temperature. Finally, we showed that this semiclassical expression allows us to test the correspondence principle for high temperatures by making $\hbar \rightarrow 0$. To conclude, one can notice that in general the characteristic function $G^{Q}(u)=$ $\operatorname{Tr}\left[e^{i u H_{\xi_{f}}} U_{\tau} e^{-i u H_{\xi_{0}}} \rho_{\beta} U_{\tau}^{\dagger}\right]$, where $U_{\tau}$ is the unitary operation resulting from a given process, can be also interpreted as a quench $G^{Q}(u)=\operatorname{Tr}\left[e^{i u\left(U_{\tau}^{\dagger} H_{\xi_{f}} U_{\tau}\right)} e^{-i u H_{\xi_{0}}} \rho_{\beta}\right]$, therefore we expect that the ideas presented in this work could also be extended to processes different from quenches [41].

The authors acknowledge Marcos Saraceno for useful discussions, and financial support from CONICET (Grants No. PIP 114-20110100048 and No. PIP 11220080100728), ANPCyT (Grants No. PICT-2013-0621 and No. PICT 20143711), and UBACyT.
[1] M. Campisi, P. Hänggi, and P. Talkner, Rev. Mod. Phys. 83, 771 (2011).

[2] J. Goold, M. Huber, A. Riera, L. del Rio, and P. Skrzypczyk, J. Phys. A 49, 143001 (2016).

[3] J. Kurchan, arXiv:cond-mat/0007360.

[4] H. Tasaki, arXiv:cond-mat/0009244.

[5] P. Talkner, E. Lutz, and P. Hänggi, Phys. Rev. E 75, 050102 (2007).

[6] C. Jarzynski, Phys. Rev. Lett. 78, 2690 (1997).

[7] G. E. Crooks, Phys. Rev. E 60, 2721 (1999).

[8] C. Jarzynski, H. T. Quan, and S. Rahav, Phys. Rev. X 5, 031038 (2015).

[9] L. Zhu, Z. Gong, B. Wu, and H. T. Quan, Phys. Rev. E 93, 062108 (2016).

[10] S. Deffner and E. Lutz, Phys. Rev. E 77, 021128 (2008).

[11] P. Talkner, P. S. Burada, and P. Hänggi, Phys. Rev. E 78, 011115 (2008).

[12] M. Campisi, Phys. Rev. E 78, 051123 (2008).

[13] S. Deffner, O. Abah, and E. Lutz, Chem. Phys. 375, 200 (2010).

[14] I. J. Ford, D. S. Minor, and S. J. Binnie, Eur. J. Phys. 33, 1789 (2012).

[15] P. Talkner, M. Morillo, J. Yi, and P. Hänggi, New J. Phys. 15, 095001 (2013).

[16] A. Silva, Phys. Rev. Lett. 101, 120603 (2008).

[17] J. Vaníček and E. J. Heller, Phys. Rev. E 68, 056208 (2003).

[18] J. Vaníček, Phys. Rev. E 70, 055201(R) (2004).

[19] J. Vaníček, Phys. Rev. E 73, 046204 (2006).

[20] M. Berry, J. Phys. A 10, 2083 (1977).

[21] A. Voros, in Stochastic Behavior in Classical and Quantum Hamiltonian Systems, edited by M.-J. Giannoni, A. Voros, and J. Zinn-Justin, Lectures Notes in Physics Vol. 93 (Springer, Berlin, 1979).

[22] G. Huber, F. Schmidt-Kaler, S. Deffner, and E. Lutz, Phys. Rev. Lett. 101, 070403 (2008).

[23] R. Dorner, S. R. Clark, L. Heaney, R. Fazio, J. Goold, and V. Vedral, Phys. Rev. Lett. 110, 230601 (2013).
[24] L. Mazzola, G. De Chiara, and M. Paternostro, Phys. Rev. Lett. 110, 230602 (2013).

[25] A. J. Roncaglia, F. Cerisola, and J. P. Paz, Phys. Rev. Lett. 113, 250601 (2014).

[26] T. B. Batalhão, A. M. Souza, L. Mazzola, R. Auccaise, R. S. Sarthour, I. S. Oliveira, J. Goold, G. De Chiara, M. Paternostro, and R. M. Serra, Phys. Rev. Lett. 113, 140601 (2014).

[27] S. An, J.-N. Zhang, M. Um, D. Lv, Y. Lu, J. Zhang, Z.-Q. Yin, H. T. Quan, and K. Kim, Nat. Phys. 11, 193 (2014).

[28] T. Gorin, T. Prosen, T. Seligman, and M. Žnidarič, Phys. Rep. 435, 33 (2006).

[29] Ph. Jacquod and C. Petitjean, Adv. Phys. 58, 67 (2009).

[30] A. Goussev, R. Jalabert, H. M. Pastawski, and D. A. Wisniacki, Scholarpedia 7, 11687 (2012).

[31] W. H. Miller, J. Chem. Phys. 53, 3578 (1970); J. Phys. Chem. 105, 2942 (2001).

[32] I. García-Mata, R. O. Vallejos, and D. A. Wisniacki, New J. Phys. 13, 103040 (2011).

[33] I. García-Mata, A. J. Roncaglia, and D. A. Wisniacki, Phil. Trans. R. Soc. A 374, 0157 (2016).

[34] D. A. Wisniacki, N. Ares, and E. G. Vergini, Phys. Rev. Lett. 104, 254101 (2010).

[35] T. Zimmermann and J. Vaníček, J. Chem. Phys. 132, 241101 (2010); T. Zimmermann, J. Ruppen, B. Li, and J. Vaníček, Int. J. Quantum Chem. 110, 2426 (2010).

[36] See Supplemental Material at http://link.aps.org/supplemental/ 10.1103/PhysRevE.95.050102 where we describe in detail the semiclassical definition of the characteristic function and how in the small $\hbar$ limit we obtain the correct work distribution.

[37] C. D. Schwieters and J. B. Delos, Phys. Rev. A 51, 1030 (1995).

[38] H. T. Quan and C. Jarzynski, Phys. Rev. E 85, 031102 (2012).

[39] E. Vergini and M. Saraceno, Phys. Rev. E 52, 2204 (1995).

[40] E. Zambrano, M. Šulc, and J. Vaníček, J. Chem. Phys. 139, 054109 (2013).

[41] I. García-Mata, A. J. Roncaglia, and D. A. Wisniacki (unpublished). 